\title{
Preface Issue 2-2016
}

\author{
Hans-Christoph Grunau ${ }^{1}$
}

(C) Deutsche Mathematiker-Vereinigung and Springer-Verlag Berlin Heidelberg 2016

The action of a group $G$ on a set $X$ is called $n$-transitive if for any two $n$-tuples of distinct elements from $X$ there is some $g \in G$ such that the action of $g$ maps the first one componentwise onto the second one. An $n$-transitive group action is sharply $n$ transitive if for any two such pairs of $n$-tuples this $g \in G$ is unique. The affine group is perhaps the best known example of a 2-transitive group: given two pairs $\left(p_{1}, p_{2}\right)$ and $\left(q_{1}, q_{1}\right)$ of distinct points in affine space, there is some affine transformation $g$ mapping $p_{i}$ to $q_{i}(i=1,2)$. The action is sharply 2-transitive if and only if the dimension of the affine space is 1 . In this example the group contains an abelian normal subgroup acting regularly, i.e. sharply 1-transitive. In her survey article "Infinite sharply multiply transitive groups" Katrin Tent explains first the known results about sharply multiply transitive group actions. For $n \geq 4$ this property is quite restrictive and the few groups that obey it have been known for a long time. Finite sharply 2and 3-transitive groups were classified by Zassenhaus in the 1930's, while the case of infinite groups has remained open since then. Katrin Tent reports on her surprising and fairly general construction of infinite sharply 2- and 3-transitive groups, which do not contain a regular abelian normal subgroup.

Perhaps, every mathematician knows floating point arithmetic as a necessary evil in numerical analysis. It is necessary because it is impossible to implement exactly all real numbers and it might become an evil because it might happen that rounding errors accumulate in such a way that the final result may no longer be reliable. When using modern and powerful computer algebra and numerical software packages one has, however, the feeling that in most cases a reasonable part of the displayed digitssay 10 of 16 - is reliable. People working in numerical analysis know how much

\section{H.-Ch. Grunau}

hans-christoph.grunau@ovgu.de

1 Institut für Analysis und Numerik, Fakultät für Mathematik, Otto-von-Guericke-Universität, Postfach 4120, 39016 Magdeburg, Germany 
effort, care and experience is needed to indeed guarantee this precision. Folkmar Bornemann's survey article on "The SIAM 100-Digit Challenge: A Decade later" revisits some particular numerical challenges and demonstrates very vividly to what extent, beside all these issues, a broad knowledge and experience is needed in what is sometimes called "pure mathematics."

The first book review of the present issue is concerned with a monograph which is dedicated also to a rather delicate numerical topic, namely numerical weather prediction. Jörn Behrens gives a brief summary and his opinion of Martin Ehrendorfer's book on "Spectral numerical weather prediction models." Andreas Knauf then reviews Scott Dumas' "The KAM story." The last book under review collects nine research lecture notes on "Moduli spaces of Riemann surfaces." These lectures were given by Minsky, Hamenstädt, Wahl, Galatius, Putman, Faber, Wolpert, Möller, and Matsumoto. This collection was edited by Benson Farb, Richard Hain, and Eduard Looijenga and is reviewed by Ulrike Tillmann. 